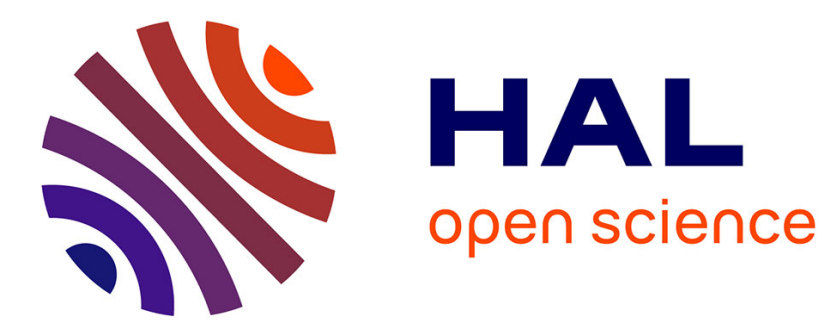

\title{
Analysis of the Network Attachment Delay of Mobile Devices in the Industrial Internet of Things
}

Rodrigo Teles Hermeto, Quentin Bramas, Antoine Gallais, Fabrice Theoleyre

\section{To cite this version:}

Rodrigo Teles Hermeto, Quentin Bramas, Antoine Gallais, Fabrice Theoleyre. Analysis of the Network Attachment Delay of Mobile Devices in the Industrial Internet of Things. International Conference on Ad Hoc Networks and Wireless (ADHOC-NOW), Oct 2019, Luxembourg, Luxembourg. pp.90-101, 10.1007/978-3-030-31831-4_7 . hal-02323215

\section{HAL Id: hal-02323215 https://hal.science/hal-02323215}

Submitted on 6 May 2020

HAL is a multi-disciplinary open access archive for the deposit and dissemination of scientific research documents, whether they are published or not. The documents may come from teaching and research institutions in France or abroad, or from public or private research centers.
L'archive ouverte pluridisciplinaire HAL, est destinée au dépôt et à la diffusion de documents scientifiques de niveau recherche, publiés ou non, émanant des établissements d'enseignement et de recherche français ou étrangers, des laboratoires publics ou privés. 


\title{
Analysis of the Network Attachment Delay of Mobile Devices in the Industrial Internet of Things
}

\author{
Rodrigo Teles Hermeto ${ }^{1}$, Quentin Bramas ${ }^{1}$, Antoine Gallais ${ }^{1,2}$, and Fabrice \\ Théoleyre ${ }^{1[0000-0002-7903-3520]}$ \\ 1 ICube Laboratory, CNRS, University of Strasbourg, France \\ teleshermeto, bramas, gallais, theoleyre@unistra.fr \\ ${ }^{2}$ FUN (Self-organizing Future Ubiquitous Network) Inria Lille - Nord Europe, France
}

\begin{abstract}
Industrial networks are typically used to monitor safetyrelated processes where high reliability and an upper bounded latency are crucial. Because of its flexibility, wireless is more and more popular, even for real-time applications. Because radio transmissions are known to be lossy, deterministic protocols have been proposed, to schedule carefully the transmissions to avoid collisions. In parallel, industrial environments now integrate mobile industrial robots to enable the Industry 4.0. Thus, the challenge consists in handling a set of mobile devices inside a static wireless network infrastructure. A mobile robot has to join the network before being able to communicate. Here, we analyze this attachment delay, comprising both the synchronization, and the negotiation of dedicated cells. In particular, since the control frames (EB and 6P) have a strong impact on the convergence, our proposed model carefully integrates the collision probability of these packets. We validate the accuracy of our model, and we analyze the impact of the different EB transmission policies on the discovery delay. Our performance evaluation demonstrates the interest of using efficiently the radio resources for beacons to handle these mobiles devices.
\end{abstract}

\section{Introduction}

Industrial networks are now widely used for many industrial applications, where high reliability and an upper bounded latency are critical. They typically rely on a costly and inflexible wired infrastructures to attend these strict requirements. In order to reduce deployment and maintenance costs, industrial networks have started to replace this legacy infrastructure with wireless sensor networks.

Due to its low-power nature, a sensor network is known to be lossy with no delivery guarantees. Thus, standards such as IEEE 802.15.4-TSCH have been released, proposing reliable mechanisms to the MAC layer in order to implement deterministic protocols. Combining the schedule of transmissions and a slow-channel hopping mechanism, a network can achieve $99.99 \%$ of end-to-end delivery rate, while upper bounding the end-to-end latency [1]. Specifically, the 
transmissions are carefully scheduled to avoid collisions, either in a distributed or a centralized manner [2].

Although mobility plays an increasingly important role for many industrial deployments [3], the IEEE 802.15.4-TSCH standard does not propose a clear approach to handle a high rate of topology changes due to the association/dissociation of mobile devices. Additionally, the slow-channel hopping mechanism introduces a new layer of complexity: a joining node has to wait for receiving the synchronization beacon on its active listening channel, delaying its association to the network. A fast association is a key factor to enable mobility over low-power wireless networks [4].

The use of mobile devices in wireless industrial networks has already been investigated in the past $[3,5,6]$. They mainly focus on proposing mechanisms to reduce the attachment delay. Indeed, discovering the network is particularly challenging in multichannel environments, since the discovering node has to find the right channel to listen to [7]. Besides, the novel device has to reserve some transmission opportunities, using control packets. Unfortunately, these control packets are prone to collisions since they are transmitted through contentionbased cells [8]. Mechanically, these collisions increase the attachment delay.

The contributions presented in this paper are as follows:

1. we propose here an analytical Markov chain to model the first association of a mobile node in a multi-hop network. We consider both the discovery of a neighboring device, and the negotiation of cells.

2. we evaluate the gain of transmitting Enhanced Beacons (EB) on multiple channels in order to reduce the synchronization delay. Using multiple channels allows to spread the load on shared cells, reducing the collision probability;

3. we quantify the impact of the network density on the discovery and negotiation time. More neighbors mean also more collisions, very prejudicial to the synchronization.

\section{Background \& related work}

We present here the most important mechanisms of IEEE 802.15.4-TSCH and $6 \mathrm{TiSCH}$, since we rely on these two standards for modeling the attachment delay of mobile nodes in networks with real-time performance.

\section{$2.1 \quad 6 \mathrm{TiSCH}$ stack}

The IEEE 802.15.4-TSCH standard has defined the TSCH mode [9], where nodes schedule the transmissions such that each application has enough transmission opportunities while avoiding collisions. The network is globally synchronized, each node maintaining the number of timeslots since the network has been created, aka. the Absolute Slot Number (ASN). A slotframe in TSCH consists in a matrix of cells of equal length, each cell being defined by a pair of timeslot and channel offsets. The schedule comprises two types of cells: 
shared cells implement a slotted-Aloha approach. For unicast packets, the absence of acknowledgment is interpreted as a collision. In that case, the transmitter triggers a random backoff value and skips the corresponding number of shared cells;

dedicated cells are allocated to interference-free transmitters to avoid any collision. This allocation may be centralized or distributed [2]. The transmitter may trigger a Clear Channel Assessment (CCA), but only to combat external interference.

6 TiSCH has defined the 6top Protocol (6P) to allocate/deallocate cells with a neighbor node [10]. By default, each schedule modification is based on a two-way handshake. The inquirer sends a request to a neighbor (e.g. preferred parent), piggybacking a list of possible cells. Then, the neighbor will acknowledge the request, selecting the cells present in the list which are also available in its schedule. When a node joins the network, it relies on shared cells to bootstrap a negotiation with its next-hop neighbor [11], since the two nodes have no common preallocated dedicated cells.

\subsection{Mobility in industrial scenarios}

Tinka et al. [12] detail a scheduling algorithm to handle a network infrastructure where all the devices are mobile. A gossip mechanism makes the schedule dissemination robust. Similarly, Vahabi et al. [13] address a mobile sink scenario. However, fully-mobile topologies make high-reliability very challenging, which jeopardizes the correct operation of many industrial applications.

A mobile node has first to discover the network, i.e. to receive an Enhanced Beacon (EB) which contains all the information for the synchronization (e.g. slotframe length, ASN, hopping sequence, etc.). However, IEEE 802.15.4-TSCH lets the schedule policy of EB unspecified, while it has a strong impact on the discovery time.

Nidawi et al. [5] propose to modify the acknowledgment packets to accelerate the discovery. Acks are grouped at the end of the slotframe, and piggyback the time that the node will keep its radio on to receive possibly new association requests. However, it requires to modify the standard.

Vogli et al. [14] consider to broadcast EB on multiple channels at once. For that purpose, they allocate exclusive timeslots for EB transmissions, with a Random Filling scheduling, where the channel offsets are selected randomly among the available ones.

Zhou et al. [15] propose rather to schedule the Enhanced Beacons to reduce the collisions, and thus, the attachment delay. However, the proposition targets more the co-existence of multiple TSCH star networks, where EBs are scheduled independently. De Guglielmo et al. [16] present a Model-based Beacon Scheduling (MBS) algorithm that minimizes the average joining time. Karalis et al. [17] propose to assign one dedicated cell for each EB, using multiple channels to avoid collisions. However, these last two approaches rely on a perfect, centralized, collision-free schedule, which makes the scheme less scalable. 
Dezfouli et al. [6] also consider the time required to negotiate dedicated cells. Indeed, being synchronized is not sufficient, the mobile device has to know when it can transmit safely its packets. For this purpose, the scheduler computes a path for each mobile device for each of its possible locations. Then, it allocates statically a collection of cells along each of these paths. However, the trajectory has to be known a priori, and it consumes much radio resource, since one single path is used at a time.

Haxhibeqiri et al. [18] focus on the handover process by employing a singlehop network with multiple gateway nodes and a centralized manager. Once a mobile node reserves dedicated cells to one gateway, the network manager instructs the others gateways to install the same cell for that node. This way, gateways have pre-allocated dedicated cells for each mobile node, reducing the handover latency. However, the proposed architecture cannot be easily extended due to deployment costs. In particular, the infrastructure requires devices with higher computational power connected through wires for the control plane.

\section{Joining time model}

We analyze here the joining time, i.e. time interval between a mobile device wakes-up, and it can start transmitting data packets through dedicated cells.

\subsection{Scenario and Assumptions}

We focus here on a network topology where the sink and a collection of relay nodes are static. Only a few devices (e.g. robots) are mobile and represent the leaves of the network infrastructure. Thus, a mobile device sends its packet to a neighboring relay node, which forwards them through a path of relays to the sink. Each static node has a collection of dedicated cells in its schedule, maintained by a scheduling function such as SF0 [11]. Thus, each relay node can forward the packets from mobile devices without any collision.

Mobile devices constitute the leaves and have to identify a single neighboring relay node to send their packets. They need to capture its Enhanced Beacons, to adjust their clock and know when are the next shared cells, to be able to transmit their first messages. After selecting a next hop, a mobile node engages a $6 \mathrm{P}$ two-way handshake [10] to reserve dedicated cells for its transmissions.

We focus on the discovery that a node has to trigger when it is unsynchronized. This procedure comprises:

synchronization: the joining node has to receive an Enhanced Beacon (EB) to synchronize itself with the network. Then, it gets the frequency hopping sequence and the shared cells for broadcast packets;

negotiation: the node has selected the source of the EB as parent, and then negotiates a set of dedicated cells to use to transmit its data packets. 


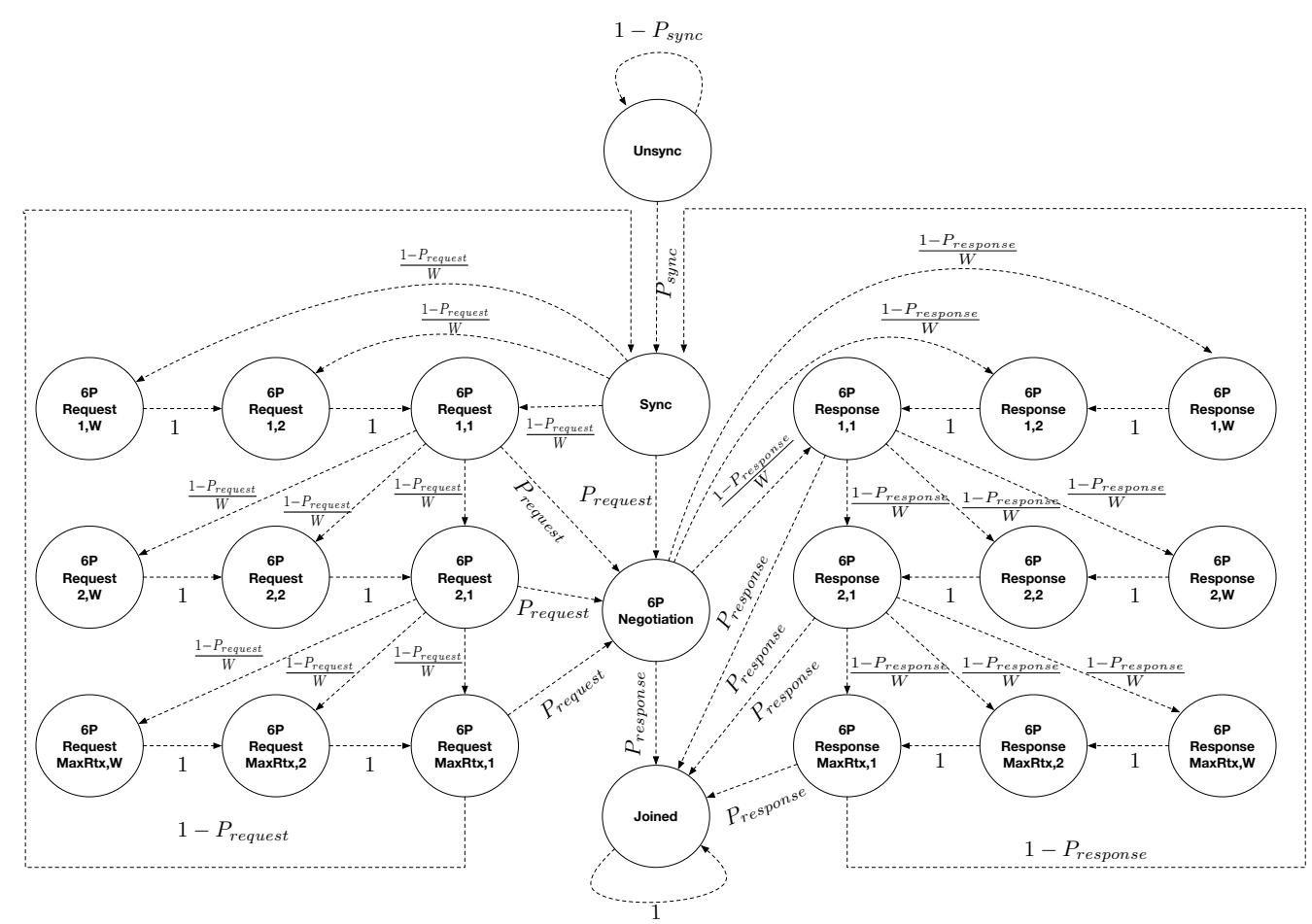

Fig. 1: Model for the association time of a joining node.

\subsection{Markov chain}

We define here a discrete time Markov chain (Fig. 1) to represent the joining process of a new (mobile) node, hereafter denoted as joining node, when it joins the network for the first time. We will detail here the different parts of our model.

\subsection{Synchronization}

The joining node is initially in the unsynchronized state, listening for EB sent by neighboring fixed nodes. In IEEE 802.15.4-TSCH, all synchronized nodes broadcast EB periodically to announce the existence of the network. We make here a distinction between the two factors that impact directly the synchronization time of the joining node: EB collision and the channel hopping mechanism.

Since IEEE 802.15.4-TSCH adopts a slotted Aloha mechanism for shared cells, the collision probability may be quite high. Indeed, an EB packet is enqueued until the next shared cell. Thus, when multiple nodes enqueue EB packets simultaneously between consecutive shared cells, their transmissions collide. In addition, because of the channel hopping characteristic, the EB is successfully received by the joining node only if the latter is listening to the right channel. 


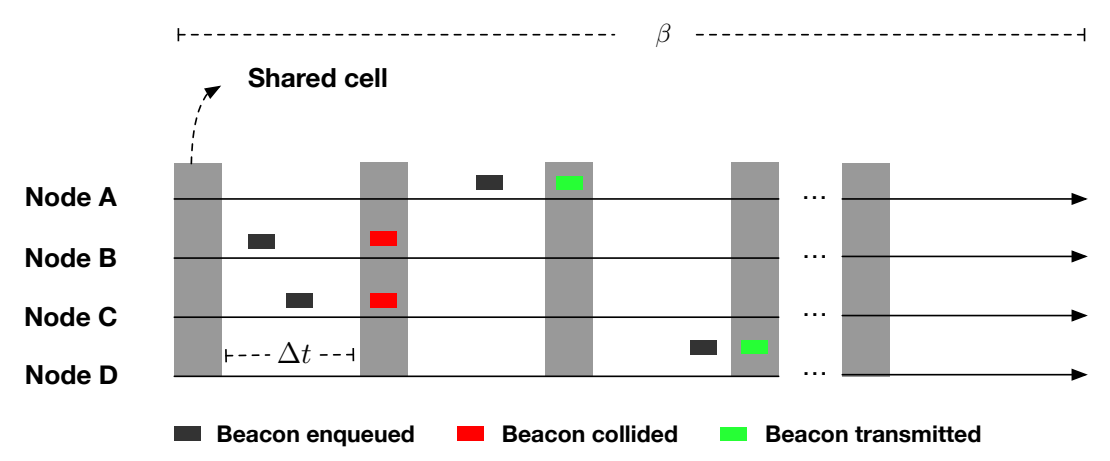

Fig. 2: Beacon queuing over the time. A collision occurs when two or more nodes enqueue simultaneously between consecutive shared cells (nodes B and C).

All nodes in a IEEE 802.15.4-TSCH network enqueue EB at the same frequency after they synchronize. To reduce the amount of collisions among EB, we consider adding jitters before EB transmissions, which represents the default behavior of OpenWSN [19]. The jitter increases the time window in which a node enqueues Enhanced Beacons. For instance, for a beacon period $\beta$ and jitter $\gamma$, the generation time of the next Enhanced Beacon will be randomly selected within the interval $[\beta-\gamma, \beta+\gamma]$.

Let us model the EB generation as a Poisson Process. Let us consider $\lambda$ as the expected number of EB queued by all nodes during a given time interval of length $L$. Let $\Delta t$ be the time between consecutive shared cells, in a such way that $L \geq \Delta t$. During the period $L$, the nodes have $\left\lfloor\frac{L}{\Delta t}\right\rfloor$ possibilities to enqueue their respective EB packets between consecutive shared cells. Since we assume that the rate $\lambda$ is constant over the time, we can compute the rate of beacons to be enqueued during any $\Delta t$ interval as:

$$
\mu=\lambda *\left\lfloor\frac{L}{\Delta t}\right\rfloor^{-1}
$$

The transmission is only successful when a single device enqueues an EB during a given $\Delta t$ interval. For instance, Figure 2 depicts two colliding transmissions (from nodes B and C). It also shows that those from A and D are successful since enqueued during different $\Delta t$ periods. From the Poisson distribution, the probability of having a single node generating an $\mathrm{EB}$ for any $\Delta t$ interval is:

$$
P_{\text {beacon }}=P(X=1)=\mu e^{-\mu}
$$

Additionally, we need to account the probability that the joining node is listening to the right channel. Since the frequency hopping sequence uses all the channels uniformly, the joining node has a uniform probability of matching the channel of the EB transmission. Thus, the probability of reception is finally:

$$
P_{\text {sync }}=P_{\text {beacon }} *\left(\frac{1}{N_{c h}}\right)
$$


where $N_{c h}$ is the number of channels.

\subsection{Negotiation}

After having received an EB, the joining node is able to synchronize itself with its parent, and to identify the shared cells to listen to. However, no bandwidth is yet available: it has to send $6 \mathrm{P}$ request packets and to wait for a confirmation before starting using dedicated cells for communication. Unfortunately, collisions are frequent in shared cells, since EB, routing control packets (i.e. DIO used by IETF RPL) and $6 \mathrm{P}$ control packets compete for the same resource.

$6 \mathrm{P}$ uses a two-way handshake mechanism: both the request and the response are subject to transmission failures. The negotiation is successful if both the request and response are transmitted without collision. Thus, we can employ here Eq. 2 with $X=0$ to compute the probability of success.

$$
P_{\text {request }}=P_{\text {response }}=e^{-\mu}
$$

In case of collision, the transmitter selects a random backoff value and skips the corresponding number of shared cells. We represent the backoff state as a 2-tuple $(r, w)$, where $r$ is the current transmission attempt and $w$ is the backoff counter. The probability of reaching any subsequent backoff state after a collision is equally likely. For all states $(i, w)$, where $w>1$, the transmitter does not try to retransmit and it transits to state $(i, w-1)$ with probability 1 . After reaching a maximum number of attempts (MaxRtx), the node discards the current packet and starts over the negotiation, i.e. go back to the sync state.

\subsection{Handover}

Since mobile devices are constantly moving around the environment, the link between the device and its point of attachment may eventually start to provide a low reliability due to the long distance between them. In that case, the mobile device has to select a more reliable relay node to forward its packets, i.e. to perform a handover. Specifically, the mobile device deallocates the cells toward its previous next hop, and negotiates novel cells with its novel relay node.

We can neglect the deallocation time, as the $6 \mathrm{P}$ packets will be transmitted without contention, i.e. dedicated cells already exist in their schedule. On the other hand, novel cells have to be negotiated with the novel relay node. Since the mobile node is already synchronized (Sync state), it can immediately engage a negotiation. Thus, we can employ Equation 4 to compute the probability that the negotiation will succeed.

\subsection{Estimating the joining time}

Since we rely on an absorbing Markov chain, we can estimate the joining time by computing the average number of steps to reach the absorbing state from the initial state Unsync. In our DTMC depicted in Figure 1, the Joined state 


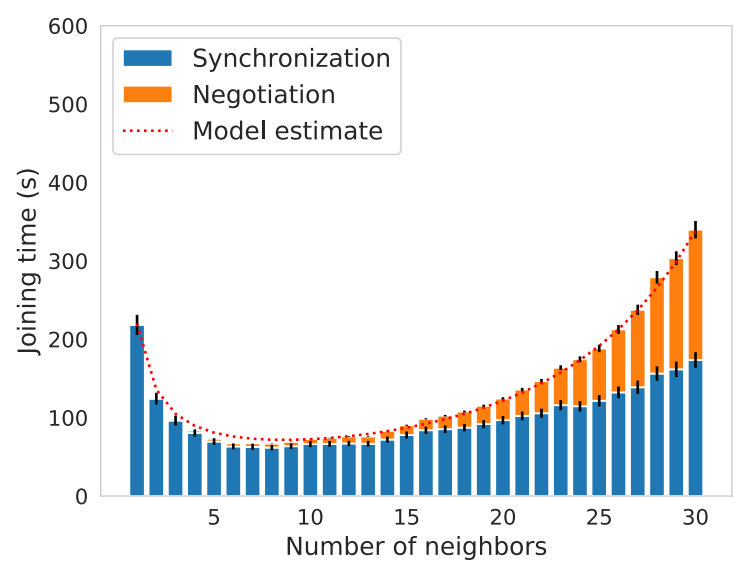

Fig. 3: Comparison of the average joining time given by the model and simulations with an EB period of $15 \mathrm{~s}$, and a jitter of $200 \mathrm{~ms}$

is the absorbing state. Every step in our model represents the interval between two consecutive shared cells (i.e. $\Delta t$ ). We rely on the Fundamental Matrix to compute the average absorbing time: i.e. number of transitions from the initial state (Unsync) to the absorbing state (Joined).

\section{Numerical Analysis}

We propose first to verify the accuracy of our DTMC model when estimating the joining time in $6 \mathrm{TiSCH}$ networks. Then, we will analyze the joining time for a joining node, as well as assessing the gain of using multiple channels for EB transmissions.

\subsection{Model validation}

We rely on simulations to validate our DTMC model. We implement a lightweight $6 \mathrm{TiSCH}$ simulator written in Python focusing exclusively on the joining procedure of a mobile node. Our simulator is freely available on GitHub

In our scenarios, we consider an existing network composed of fixed nodes (i.e. the infrastructure) and one joining node. The fixed nodes broadcast EB and DIO regularly during shared cells. For sake of simplicity, we assume that the infrastructure has enough bandwidth to accommodate the novel flows. Thus, only the joining node and its point of attachment have to negotiate dedicated cells. Additionally, we assume perfect links conditions. Thus, collisions are the only causes of packet drops.

We employ a slotframe composed of 101 timeslots, and 26 channel offsets, with two shared cells placed uniformly in the slotframe. The joining node selects

\footnotetext{
${ }^{\ddagger}$ https://github.com/rodrigoth/Simulator/tree/optimized
} 


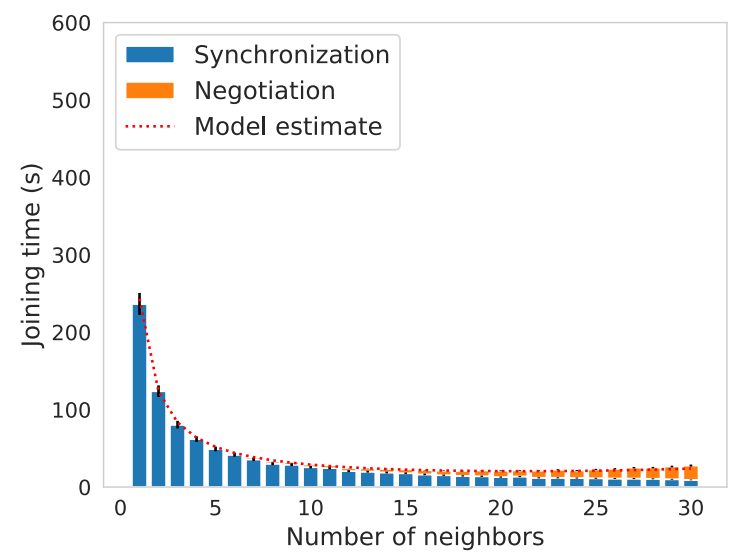

Fig. 4: Impact of the multi-channel EB broadcasting on the joining time.

randomly one channel to listen for EB. All nodes in the infrastructure enqueue EB and DIO every 15 seconds in average, considering a jitter of $200 \mathrm{~ms}$. We plot systematically the $95 \%$ confidence intervals.

The comparison between our analytical model and simulation results are depicted in Figure 3. We perform 1,000 repetitions for each number of neighbors to make our results more representative. We observe that the analytical values fit very well the simulation results. As expected, with few nodes, the synchronization takes longer, since the joining node has a smaller probability to receive a valid EB. On the other hand, the negotiation is fast, since there are less competition in the shared cells. Increasing the number of neighbors improves the joining time to a certain extent (i.e. 9 neighbors in our scenario). For higher values, the probability of collision increases impacting both the synchronization and negotiation times. Thus, the joining time presents an exponential growth.

\subsection{Multi-channel EB to reduce the attachment delay}

By definition, all transmissions in shared cells occur exclusively in a single channel offset. Therefore, the probability of collision increases, since only one cell is used for transmissions. We propose to assess the gain of the Random Filling approach [14] to transmit EB on multiple channels, and thus, to reduce the synchronization time.

We redefine Equation 3 to account simultaneous transmissions on different channels. Now, on each channel, EB arrival follows a Poisson Process of parameter $\mu / N_{c h}$, since the EB are uniformly distributed on all $N_{c h}$ channels. Hence,the probability that only one EB is transmitted on the channel that the joining node is listening to is:

$$
P_{\text {sync }}=\left(\frac{\mu}{N_{c h}}\right) e^{\left(-\frac{\mu}{N_{c h}}\right)}
$$




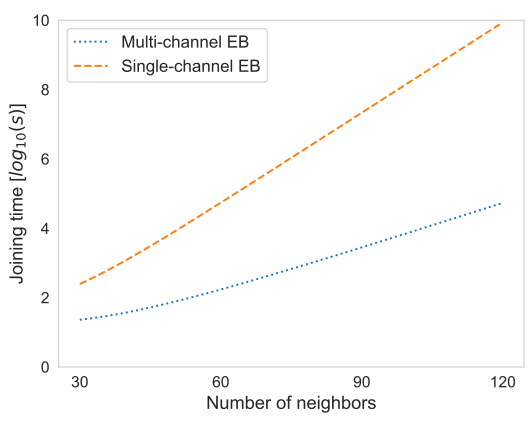

(a) EB period: $15 \mathrm{~s}$

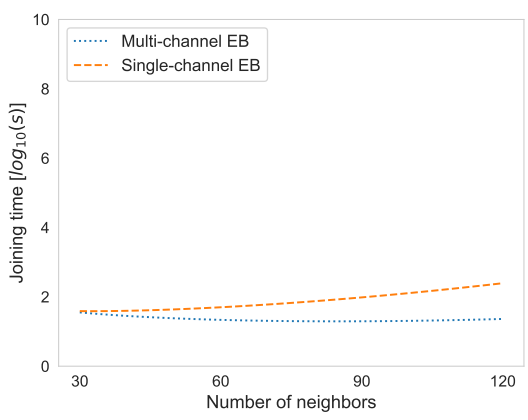

(c) EB period: $60 \mathrm{~s}$

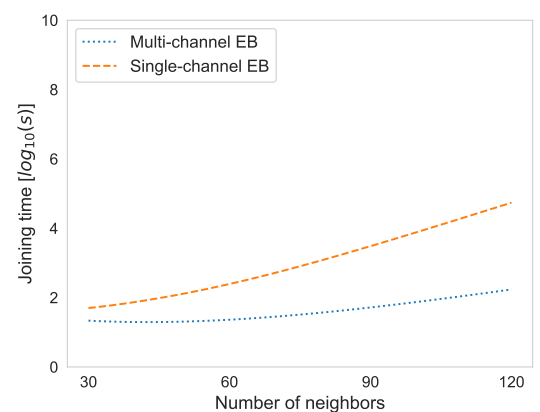

(b) EB period: 30s

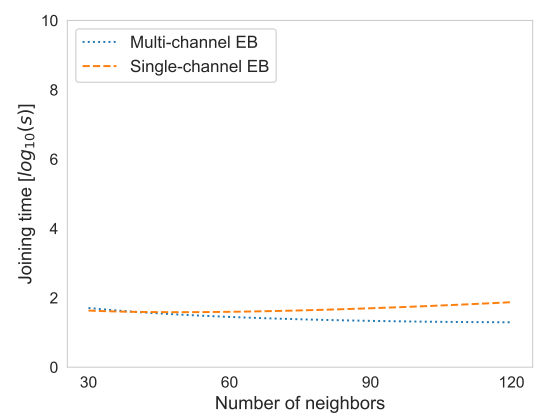

(d) EB period: $90 \mathrm{~s}$

Fig. 5: Impact of the EB period on different network densities.

Figure 4 highlights the gain of broadcasting EB on multiple channels. The synchronization time decreases heavily compared to the single channel case depicted in Figure 3. In a general way, we can expect less EB arrivals, but the EB are now transmitted on multiple channels simultaneously. Thus, the probability that the joining node is listening to any of the transmitted channels increases. In addition, spreading EB on multiple channel impacts directly the negotiation time, since the EB and the $6 \mathrm{P}$ packets are transmitted mostly on different channels. We can now accommodate much larger densities with a very reasonable attachment delay.

\subsection{Large scale performance}

Finally, we analyze the impact of the EB period on the joining time with different densities. For the sack of simplicity, we consider that the EB and DIO are transmitted at the same frequency.

Figure 5 compares the joining time when EB are transmitted on single vs. multiple channels. As expected the EB frequency has a crucial importance on the 
joining time on large scale deployments. However, shorter EB periods increase the probability of collisions when using a single channel. Using multiple channels allows to spread the load, and thus reduces significantly the collision probability.

We can note that the optimal EB period, minimizing the joining time, depends on the density. More nodes mean a larger number of EB transmissions, and thus a larger optimal EB period.

\section{Conclusions \& Perspectives}

In this work, we focused on the joining time of a mobile node when it joins the network for the first time. Mobile devices have to fast attach to the network, and reserve some resources for their critical flows. We modeled the joining process using a Discrete Time Markov Chain. Our model takes into account both the synchronization and negotiation times in $6 \mathrm{TiSCH}$ networks.

Our simulations demonstrate the accuracy of our DTMC model to estimate finely the synchronization and negotiation time. Obviously, dense networks mean a larger number of collisions, which impact very negatively the synchronization time. Even worse, negotiating dedicated cells is also very expensive, since the collision rate for control packets is very high. We also use our DTMC model to assess the gain of using multiple channels. By spreading the EB on the different channels, the collision rate is significantly reduced, improving the scalability.

As a future work, we plan to propose an handover scheme, so that a mobile device can maintain several next hops, to avoid dropping data packets. In particular, we have to reduce the negotiation time, when a novel relay node is identified. Recent approaches based on autonomous scheduling, such as MSF [20], seem promising to reduce the contention, particularly when a large number of mobile devices has to attach to the network simultaneously.

\section{References}

1. Simon Duquennoy, Beshr Al Nahas, Olaf Landsiedel, and Thomas Watteyne. Orchestra: Robust Mesh Networks Through Autonomously Scheduled TSCH. In SenSys, pages 337-350. ACM, 2015.

2. R. T. Hermeto, A. Gallais, and F. Theoleyre. Scheduling for IEEE 802.15.4-TSCH and slow channel hopping MAC in low power industrial wireless networks: A survey. Computer Communications, 114:84 - 105, 2017.

3. R. Silva, J. S. Silva, and F. Boavida. Infrastructure-supported mobility in wireless sensor networks - a case study. In IEEE International Conference on Industrial Technology (ICIT), pages 1895-1900, March 2015.

4. Pranesh Sthapit, Yeon-Sang Choi, Goo-Rak Kwon, SS Hwang, and JY Pyun. A fast association scheme over IEEE 802.15. 4 based mobile sensor network. Proc. $I C W M C, 2013$.

5. Y. Al-Nidawi and A. H. Kemp. Mobility aware framework for timeslotted channel hopping ieee 802.15.4e sensor networks. IEEE Sensors Journal, 15(12):7112-7125, Dec 2015. 
6. B. Dezfouli, M. Radi, and O. Chipara. Real-time communication in low-power mobile wireless networks. In 13th IEEE Annual Consumer Communications Networking Conference (CCNC), pages 680-686, Jan 2016.

7. N. Karowski, A. C. Viana, and A. Wolisz. Optimized Asynchronous Multichannel Discovery of IEEE 802.15.4-Based Wireless Personal Area Networks. IEEE Transactions on Mobile Computing, 12(10):1972-1985, Oct 2013.

8. Fabrice Theoleyre and Georgios Z Papadopoulos. Experimental validation of a distributed self-configured 6 tisch with traffic isolation in low power lossy networks. In International Conference on Modeling, Analysis and Simulation of Wireless and Mobile Systems (MSWiM), pages 102-110. ACM, 2016.

9. IEEE Standard for Low-Rate Wireless Networks. IEEE Std 802.15.4-2015 (Revision of IEEE Std 802.15.4-2011), April 2016.

10. Q. Wang, X. Vilajosana, and T. Watteyne. 6top Protocol (6P). draft, IETF, October 2017. draft-ietf-6tisch-6top-protocol-09.

11. Diego Dujovne, LA Grieco, Maria Rita Palattella, and Nicola Accettura. 6TiSCH 6top Scheduling Function Zero (SF0). Internet-draft, IETF, 2016. draft-ietf-6tisch6top-sf0-00.

12. Andrew Tinka, Thomas Watteyne, Kristofer S. J. Pister, and Alexandre M. Bayen. A decentralized scheduling algorithm for time synchronized channel hopping. EAI Endorsed Transactions on Mobile Communications and Applications, 1(1), 92011.

13. M. Vahabi, H. R. Faragardi, and H. Fotouhi. An analytical model for deploying mobile sinks in industrial internet of things. In IEEE Wireless Communications and Networking Conference Workshops (WCNCW), pages 155-160, April 2018.

14. Elvis Vogli, Giuseppe Ribezzo, L Alfredo Grieco, and Gennaro Boggia. Fast network joining algorithms in industrial IEEE 802.15.4 deployments. Ad Hoc Networks, 69:65-75, 2018.

15. Mengchuan Zou, Jia-Liang Lu, Fan Yang, Mathilde Malaspina, Fabrice Theoleyre, and Min-You Wu. Distributed scheduling of enhanced beacons for ieee802.15.4tsch body area networks. In International Conference on Ad-Hoc Networks and Wireless (ADHOC-NOW), pages 3-16, 2016.

16. D. De Guglielmo, S. Brienza, and G. Anastasi. A Model-based Beacon Scheduling algorithm for IEEE 802.15.4e TSCH networks. In International Symposium on A World of Wireless, Mobile and Multimedia Networks (WoWMoM), pages 1-9. IEEE, June 2016.

17. Apostolos Karalis, Dimitrios Zorbas, and Christos Douligeris. Collision-Free Broadcast Methods for IEEE 802.15.4-TSCH Networks Formation. In International Conference on Modeling, Analysis and Simulation of Wireless and Mobile Systems (MSWiM), pages 91-98. ACM, 2018.

18. Jetmir Haxhibeqiri, Abdulkadir Karaağaç, Ingrid Moerman, and Jeroen Hoebeke. Seamless roaming and guaranteed communication using a synchronized single-hop multi-gateway 802.15. 4e tsch network. Ad Hoc Networks, 86:1-14, 2019.

19. Thomas Watteyne, Xavier Vilajosana, Branko Kerkez, Fabien Chraim, Kevin Weekly, Qin Wang, Steven Glaser, and Kris Pister. OpenWSN: a standards-based low-power wireless development environment. Transactions on Emerging Telecommunications Technologies, 23(5):480-493, 2012.

20. T. Chang, M. Vucinic, X. Vilajosana, S. Duquennoy, and D. Dujovne. 6TiSCH Minimal Scheduling Function (MSF). Internet-draft, IETF, April 2019. draftchang-6tisch-msf-03. 